\title{
On neutral impulsive stochastic differential equations with Poisson jumps
}

\author{
Anguraj Annamalai', Banupriya Kandasamy', Dumitru Baleanu ${ }^{2,3 *}$ and Vinodkumar Arumugam ${ }^{4}$
}

"Correspondence:

dumitru@cankaya.edu.tr

${ }^{2}$ Department of Mathematics, Cankaya University, Ankara, Turkey

${ }^{3}$ Institute of Space Sciences, Magurele-Bucharest, Romania Full list of author information is available at the end of the article

\begin{abstract}
We study the results of existence and continuous dependence on neutral impulsive stochastic differential equations with Poisson jumps. We have also created some conditions confirming exponential stability.
\end{abstract}

Keywords: Stochastic differential equations; Contraction mapping; Continuous dependence exponential stability; Poisson process; Impulsive system

\section{Introduction}

Stochastic differential equations have been investigated as mathematical models to describe the dynamical behavior of real life phenomena. It is essential to take into account the environmental disturbances as well as the time delay while constructing realistic models in the area of engineering, biology, etc. Neutral functional differential equations have been introduced in [11] for the deterministic case. Neutral stochastic functional differential equations (NSFDEs) have been initiated in [12] and their usage in aeroelasticity was pointed out. In the last few decades several studies on quantitative and qualitative properties of NSFDEs were carried out (see $[4,5,20]$ and the references therein).

Impulsive differential equations thrive to be a promising area and have gained much attention among the researchers due to their potential application in various fields such as orbital transfer of satellite, dosage supply in pharmacokinetics, etc. It is worth mentioning that many real world systems are subjected to stochastic abrupt changes, and therefore it is necessary to investigate them using impulsive stochastic functional differential equations. Few works have been reported in the study of NSFDEs with impulsive effects, refer to [1, $2,18]$.

Moreover, many practical systems (such as sudden price variations (jumps) due to market crashes, earthquakes, hurricanes, epidemics, and so on) may undergo some jump type stochastic perturbations. The sample paths of such systems are not continuous. Therefore, it is more appropriate to consider stochastic processes with jumps to describe such models. In general, these jump models are derived from Poisson random measure. The sample paths of such systems are right continuous and possess left limits. Recently, many researchers have been focusing their attention towards the theory and applications of NSFDEs with Poisson jumps. To be more precise, existence and stability results on NSFDEs with jump process can be found in $[3,4,6,8,14,17,19,21,23]$ and the references therein. Particularly, Boufoussi and Hajji [4] investigated successive approximation

(c) The Author(s) 2018. This article is distributed under the terms of the Creative Commons Attribution 4.0 International License (http://creativecommons.org/licenses/by/4.0/), which permits unrestricted use, distribution, and reproduction in any medium, provided you give appropriate credit to the original author(s) and the source, provide a link to the Creative Commons license, and indicate if changes were made. 
of NSFDEs with jumps. Subsequently, SDEs with Poisson jumps were established by few authors; for example, Wang et al. [21] studied them under a local non-Lipschitz condition, Cui and Yan [8] investigated them for the case of infinite delay. Chen [6, 7] studied the exponential stability by establishing impulsive integral inequality. Further, we refer [10, 15, $19,24]$ to investigate the exponential stability. The purpose of this manuscript is to study the impulsive NSFDEs driven by Poisson jumps.

This paper comprises five sections. Section 1 becomes the introduction. We recollect some basic concepts and preliminaries briefly in Sect. 2. Section 3 focuses on the study of sufficient conditions for the existence and uniqueness of mild solution to NSFDEs with impulses and Poisson process by the contraction mapping principle. The continuous dependence result is proposed in Sect. 4. Section 5 involves the results of exponential stability of mild solution by using impulsive integral inequality.

\section{Preliminaries}

Let $X$ and $Y$ be the separable Hilbert spaces and $L(Y, X)$ be the space of bounded linear operators from $Y$ into $X$. Consider a complete probability space $(\Omega, B, \mathbb{P})$ in which $B$ is a complete $\sigma$-algebra generated by $\left\{B_{t}\right\}_{t \geq 0}$, an increasing right continuous family. Assume a $Y$-valued $Q$-Wiener process $\{W(t): t \geq 0\}$ with respect to $\left\{B_{t}\right\}_{t \geq 0}$. Here $Q$ indicates the trace class covariance and positive self-adjoint operator on $Y$, that is,

$$
E\langle W(t), x\rangle_{Y}\langle W(s), y\rangle_{Y}=(t \wedge s)\langle Q x, y\rangle, \quad \text { for all } x, y \in Y
$$

Let $Y_{0}=Q^{1 / 2}(Y)$, which is a Hilbert subspace of $Y$ with $\langle u, v\rangle_{Y_{0}}=\left\langle Q^{-1 / 2} v\right\rangle_{Y}$. Let

$$
\langle W(t), e\rangle=\sum_{n=1}^{\infty} \sqrt{\lambda_{i}}\left\langle e_{i}, e\right\rangle \beta_{i}(t), \quad e \in Y
$$

where $\left\{e_{i}\right\}_{i \geq 1}$ is a complete orthonormal system which belongs to $Y$, and $Q e_{i}=\lambda_{i} e_{i}, i=$ $1,2, \ldots$, where $\lambda_{i}$ is a bounded sequence of positive real numbers and $\left\{B_{t}\right\}$ are independent Brownian motions.

Now, consider the impulsive NSFDE driven by Poisson jumps of the form

$$
\begin{aligned}
& \begin{array}{l}
d\left[x(t)+g\left(t, x_{t}\right)\right]=\left[A x(t)+f\left(t, x_{t}\right)\right] d t+\sigma\left(t, x_{t}\right) d W(t) \\
\quad+\int_{\mathcal{U}} h\left(t, x_{t}, u\right) \tilde{N}(d t, d u), \quad 0 \leq t \leq T, t \neq t_{j},
\end{array} \\
& \begin{aligned}
\Delta x\left(t_{j}\right)=x\left(t_{j}+\right)-x\left(t_{j}-\right)=I_{j}\left(x\left(t_{j}\right)\right), \quad t=t_{j}, j=1,2, \ldots, \\
x(t)=\phi(t), \quad-\tau \leq t \leq 0,
\end{aligned}
\end{aligned}
$$

where $f, g:[0,+\infty) \times X \rightarrow X, \sigma:[0, \infty) \times X \rightarrow \mathcal{L}_{2}^{0}(Y, X), h=[0, \infty) \times X \times \mathcal{U} \rightarrow X, I_{j}: X \rightarrow$ $X$, and are defined later. The space $\mathcal{L}_{2}^{0}(Y, X)$ contains all Q-Hilbert-Schmidt operators from $Y$ into $X$ with the norm $\|\zeta\|_{\mathcal{L}_{2}^{0}}^{2}:=\operatorname{tr}\left(\zeta Q \zeta^{*}\right)$, where $\zeta \in \mathcal{L}(Y, X)$.

Let $D((-\infty, 0], X)$ be the phase space with $\|\phi\|_{t}=\sup _{-\infty<\theta<0}|\phi(\theta)|$ and $D_{B_{0}}^{b}((-\infty, 0], X)$ indicates the family of almost surely bounded, $B_{0}$-measurable square integrable random variables with values in $X$. Consider the Banach space $\mathcal{B}_{T}=\mathcal{B}_{T}\left((-\infty, T], L_{2}\right)$, the family of 
all $B_{T}$-adapted processes $\phi(t, w)$ which are càdlàg (right continuous and left limit exists) in $t$ for a.e., for $w \in \Omega$

$$
\|\phi\|_{\mathcal{B}_{T}}=\left(\sup _{0 \leq t \leq T} E\|\phi\|_{t}^{2}\right)^{1 / 2}, \quad \phi \in \mathcal{B}_{T} .
$$

The counting measure of stationary Poisson process $\left(p_{t}\right)_{t>0}$ is denoted by $N(t, d u)$ and $\hat{N}(t, A)=\mathbb{E}(N(t, A))=t v(A)$ for $A \in \mathcal{E}$, where $v$ is the characteristic measure. The Poisson martingale measure is defined as $\widetilde{N}(t, d u)=N(t, d u)-t v(d u)$, generated by $p_{t}$.

The impulsive moments $t_{j}$ satisfy $0<t_{1}<t_{2}, \ldots, \lim _{j \rightarrow \infty} t_{j}=\infty, \Delta x\left(t_{j}\right)=x\left(t_{j}^{+}\right)-x\left(t_{j}^{-}\right)$, where $\Delta x\left(t_{j}\right)$ indicates the jump at time $t_{j}$ in the state $x$ with $I_{j}$ defining the size of the jump and $x\left(t_{j}^{-}\right)$and $x\left(t_{j}^{+}\right)$are respectively the left and the right limits at $t_{j}$ of $x(t)$.

Here $A: D(A) \rightarrow X$ is the infinitesimal generator of an analytic semigroup $(S(t))_{t \geq 0}$ of bounded linear operators on $X$ satisfying the usual conditions; for details, refer to [16] and [9].

Lemma 2.1 ([16]) If $0 \leq \alpha \leq 1$, then $X_{\alpha}$ is a Banach space and there exists $M_{\alpha}>0$ such that

$$
\left\|(-A)^{\alpha} S(t)\right\| \leq \frac{M_{\alpha}}{t^{\alpha}} e^{-\lambda t}, \quad t \geq 0, \text { and } \lambda>0
$$

Lemma 2.2 (Burkholder's inequality [9]) If $\phi(t), t \geq 0$ is an $\mathcal{L}_{2}^{0}$-valued predictable process and $W_{A}^{\phi}=\int_{0}^{t} S(t-s) \phi(s) d W(s), t \in[0, T]$. Then, for any arbitrary $p>2$, there exists a constant $c(p, T)>0$ such that

$$
\mathbb{E} \sup _{t \leq T}\left|W_{A}^{\phi}\right|^{p} \leq c(p ; T) \sup _{t \leq T}\|S(t)\|^{p} \mathbb{E} \int_{0}^{t}\|\phi(s)\|^{p} d s
$$

Moreover, if $\mathbb{E} \int_{0}^{t}\|\phi(s)\|^{p} d s<+\infty$, then there exists a continuous version of the process $\left\{W_{A}^{\phi}: t \geq 0\right\}$. If $(S(t))_{t \geq 0}$ is a contraction semigroup, then the above result is true for $p \geq 2$.

Lemma $2.3([22])$ Let $E(t):[-\tau,+\infty) \rightarrow[0,+\infty)$ be a function and if there exists some constant $\gamma>0, \alpha_{j}(j=1,2,3)$ and $\beta_{i}(i=1,2,3)$ satisfy

$$
E(t) \leq \alpha_{1} e^{-\gamma t} \quad \text { for } t \in[-\tau, 0]
$$

and

$$
\begin{aligned}
E(t) \leq & \alpha_{1} e^{-\gamma t}+\alpha_{2} \sup _{\theta \in[-\tau, 0]} E(t+\theta)+\alpha_{3} \int_{0}^{t} e^{-\gamma(t-s)} \sup _{\theta \in[-\tau, 0]} E(t+\theta) d s \\
& +\sum_{t_{i}<t} \beta_{i} e^{-\gamma(t-s)} E\left(t_{i}^{-}\right) \quad \text { for } t \geq 0 .
\end{aligned}
$$

If $\alpha_{2}+\frac{\alpha_{3}}{\gamma}+\sum_{i=1}^{+\infty} \beta_{i}<1$, then $E(t) \leq M e^{-\mu t}$ for $t \geq-\tau, \mu>0$ denotes the unique solution to the algebraic equation: $\alpha_{2}+\frac{\alpha_{3}}{\gamma-\mu} e^{\mu \tau}+\sum_{i=1}^{+\infty} \beta_{i}=1$ and $M=\max \left\{\frac{\alpha_{1}(\gamma-\mu)}{\alpha_{3} e^{\mu \tau}}, \alpha_{1}\right\}$. 


\section{Existence and uniqueness}

Suppose $0 \in \rho(A)$ and from Lemma 2.1, for the constants $M, M_{1-\beta},\|S(t)\| \leq M$ and $\left\|(-A)^{1-\beta} S(t)\right\| \leq \frac{M_{1-\beta}}{t^{1-\beta}}$ for every $t \in[0, T]$.

Definition 3.1 If $x:[-\tau, T] \rightarrow X$ is a stochastic process and

(i) $x(t)$ is measurable and $F_{t}$ adapted for all $-\tau \leq t \leq T$;

(ii) $x(t)$ has càdlàg paths almost surely;

(iii) $x(t)=S(t)(\phi(0)+g(0, \phi))-g\left(t, x_{t}\right)-\int_{0}^{t} A S(t-s) g\left(s, x_{s}\right) d s+\int_{0}^{t} S(t-s) f\left(s, x_{s}\right) d s+$ $\int_{0}^{t} S(t-s) \sigma\left(s, x_{s}\right) d W s+\int_{0}^{t} \int_{\mathcal{U}} S(t-s) h\left(s, x_{s}, u\right) \tilde{N}(d s, d u)+\sum_{0<t_{k}<t} S\left(t-t_{k}\right) I_{j}\left(x\left(t_{j}\right)\right)$ if $t \in[0, T]$

(iv) $x(t)=\phi(t),-\tau \leq t \leq 0$.

then $x$ is said to be the mild solution of Eqs. (2.1)-(2.3) on $[-\tau, T]$.

\section{Assumptions}

$\left(\mathrm{A}_{1}\right) f(t, \cdot), \sigma(t, \cdot)$, and $h(t, \cdot)$ satisfy the following Lipschitz conditions for all $t \in[0, T]$ and $x, y \in X$ :

(1a) $\left\|f\left(t, x_{t}\right)-f\left(t, y_{t}\right)\right\|^{2} \leq C_{f}^{2}\|x-y\|_{t}^{2}$;

(1b) $\left\|\sigma\left(t, x_{t}\right)-\sigma\left(t, y_{t}\right)\right\|^{2} \leq C_{\sigma}^{2}\|x-y\|_{t}^{2}$;

(1c) (i) $\int_{\mathcal{U}}\left\|h\left(t, x_{t}, u\right)-h\left(t, y_{t}, u\right)\right\|^{2} v(d u) \vee\left(\left(\int_{\mathcal{U}}\left\|h\left(t, x_{t}, u\right)-h\left(t, y_{t}, u\right)\right\|^{4} v(d u)\right)^{1 / 2} \leq\right.$ $C_{h} \int_{0}^{t}\|x-y\|_{t}^{2}$

(ii) $\left(\int_{\mathcal{U}}\left\|h\left(t, x_{t}, u\right)\right\|^{4} v(d u)\right)^{1 / 2} \leq C_{h}\|x\|_{t}^{2} d s$;

for some positive constants $C_{f}, C_{\sigma}, C_{h}$. We further assume that, for $t \geq 0$ and $u \in \mathcal{U}$, $f(t, 0) \vee \sigma(t, 0) \vee h(t, 0, u)=\kappa_{0}$, where $\kappa_{0}>0$ is a constant.

$\left(\mathrm{A}_{2}\right)$ The function $g$ is $X_{\beta}$-valued and satisfies

(2a) $\left\|(-A)^{-\beta}\right\| C_{g}<1$ and $g(t, 0)=0$, where the constants $\frac{1}{2}<\beta<1, C_{g}>0$.

(2b) $\left\|(-A)^{\beta} g\left(t, x_{t}\right)-(-A)^{\beta} g\left(t, y_{t}\right)\right\|^{2} \leq C_{g}^{2}\|x-y\|_{t}^{2}$ for all $t \in[0, T]$ and $x, y \in X$.

$\left(\mathrm{A}_{3}\right)$ The function $(-A)^{\beta} g$ is continuous in the quadratic mean sense:

$$
\lim _{t \rightarrow s} \mathbb{E}\left\|(-A)^{\beta}\left(g\left(t, x_{t}\right)-g\left(t, x_{s}\right)\right)\right\|^{2}=0
$$

(A $\left.\mathrm{A}_{4}\right)$ The function $I_{j} \in C(X, X)$ for all $x, y \in X,\left\|I_{j}\left(x\left(t_{j}\right)\right)-I_{j}\left(y\left(t_{j}\right)\right)\right\|^{2} \leq q_{j}^{2}\|x-y\|_{t}^{2}$, where $q_{j}$ is a constant and $j=1,2, \ldots$.

Theorem 3.1 Suppose that $\left(\mathrm{A}_{1}\right)-\left(\mathrm{A}_{4}\right)$ hold. Then, for all $T>0$, system (2.1)-(2.3) has a unique mild solution on $[-\tau, T]$ provided that

$$
\frac{5 M^{2} \sum_{j=1}^{\infty} q_{j}^{2}}{(1-k)^{2}}<1
$$

where $k=C_{g}\left\|(-A)^{-\beta}\right\|$.

Proof Define an operator $\pi: \mathcal{B}_{T} \rightarrow \mathcal{B}_{T}$ by

$$
\begin{aligned}
\pi(x(t))= & S(t)(\phi(0)+g(0, \phi))-g\left(t, x_{t}\right) \\
& -\int_{0}^{t} A S(t-s) g\left(s, x_{s}\right) d s+\int_{0}^{t} S(t-s) f\left(s, x_{s}\right) d s
\end{aligned}
$$




$$
\begin{aligned}
& +\int_{0}^{t} S(t-s) \sigma\left(s, x_{s}\right) d W(s) \\
& +\int_{0}^{t} \int_{\mathcal{U}} S(t-s) h\left(s, x_{s}, u\right) \tilde{N}(d s, d u) \\
& +\sum_{0<t_{j}<t} S\left(t-t_{j}\right) I_{j}\left(x\left(t_{j}\right)\right) \quad \text { for } t \in[0, T]
\end{aligned}
$$

and

$$
\pi(x(t))=\phi(t) \quad \text { for } t \in[-\tau, T] .
$$

Now, to prove the existence of mild solutions of (2.1)-(2.3), it is sufficient to show that $\pi$ has a fixed point.

Step (i): First, we verify that $t \rightarrow \pi(x(t))$ is càdlàg on $[0, T]$.

Let $|h|$ be small enough, for $x \in \mathcal{B}_{T}$ and $0<t<T$, we get

$$
\begin{aligned}
& \|\pi(x(t+h))-\pi(x(t))\|^{2} \\
& \leq \|[S(t+h)-S(t)][\phi(0)+g(0, \phi)]-\left[g\left(t+h, x_{t+h}\right)-g\left(t, x_{t}\right)\right] \\
& \quad-\left[\int_{0}^{t} A[S(t+h-s)-S(t-s)] g\left(s, x_{s}\right) d s+\int_{t}^{t+h} A S(t+h-s) g\left(s, x_{s}\right) d s\right] \\
& \quad+\int_{0}^{t}[S(t+h-s)-S(t-s)] f\left(s, x_{s}\right) d s+\int_{t}^{t+h} S(t+h-s) f\left(s, x_{s}\right) d s \\
& \quad+\int_{0}^{t}[S(t+h-s)-S(t-s)] \sigma\left(s, x_{s}\right) d W(s)+\int_{t}^{t+h} S(t+h-s) \sigma\left(s, x_{s}\right) d W(s) \\
& \quad+\int_{0}^{t} \int_{\mathcal{U}}[S(t+h-s)-S(t-s)] h\left(s, x_{s}, u\right) \tilde{N}(d s, d u) \\
& \quad+\int_{t}^{t+h} \int_{\mathcal{U}} S(t+h-s) h\left(s, x_{s}, u\right) \tilde{N}(d s, d u) \\
& \quad+\sum_{0<t_{j}<t}\left[S\left(t+h-t_{j}\right)-S\left(t-t_{j}\right)\right] I_{j}\left(x\left(t_{j}\right)\right)+\sum_{t<t_{j}<t+h} S\left(t+h-t_{j}\right) I_{j}\left(x\left(t_{j}\right)\right) \|^{2} \\
& \|\pi(x(t+h))-\pi(x(t))\|^{2} \\
& \leq
\end{aligned}
$$

Then employing the Lebesgue dominated theorem and the strong continuity of $S(t)$ implies that

$$
\lim _{h \rightarrow 0}\|S(t+h)-S(t)\|^{2} \mathbb{E}\|[\phi(0)+g(0, \phi)]\|^{2} \rightarrow 0 .
$$

Next, it is well known that $(-A)^{-\beta}$ is bounded,

$$
\mathbb{E}\left\|F_{1}(t+h)-F_{1}(t)\right\|^{2} \leq\left\|(-A)^{-\beta}\right\|^{2} \mathbb{E}\left\|(-A)^{\beta} g\left(t+h, x_{t+h}\right)-(-A)^{\beta} g\left(t, x_{t}\right)\right\|^{2} .
$$


By assumption $\left(\mathrm{A}_{3}\right)$, we obtain that $\lim _{h \rightarrow 0} \mathbb{E}\left\|F_{1}(t+h)-F_{1}(t)\right\|^{2} \rightarrow 0$. Then, for the term $F_{2}$, applying $\left(\mathrm{A}_{1}\right)$, Hölder's inequality, and the Lebesgue dominated theorem, we obtain

$$
\begin{aligned}
\mathbb{E}\left\|F_{2}(t+h)-F_{2}(t)\right\|^{2} \leq & 2 \mathbb{E}\left\|\int_{0}^{t}[S(t+h-s)-S(t-s)](-A)^{1-\beta}(-A)^{\beta} g\left(s, x_{s}\right) d s\right\|^{2} \\
& +2 \mathbb{E}\left\|\int_{t}^{t+h} S(t+h-s)(-A)^{1-\beta}(-A)^{\beta} g\left(s, x_{s}\right) d s\right\|^{2} \\
\leq & 2 C_{g}^{2} \cdot t \int_{0}^{t}\|S(t+h-s)-S(t-s)\|^{2}\left\|(-A)^{1-\beta}\right\|^{2} \mathbb{E}\|x\|_{s}^{2} d s \\
& +2 C_{g}^{2} \cdot h \int_{t}^{t+h}\|S(t+h-s)\|^{2}\left\|(-A)^{1-\beta}\right\|^{2} \mathbb{E}\|x\|_{s}^{2} d s \\
\rightarrow & 0 \quad \text { as }|h| \rightarrow 0 .
\end{aligned}
$$

A similar computation gives us $\mathbb{E}\left\|F_{3}(t+h)-F_{3}(t)\right\|^{2} \rightarrow 0$ as $|h| \rightarrow 0$.

Further, using Lemma 2.2 and Hölder's inequality, we get

$$
\begin{aligned}
\mathbb{E}\left\|F_{4}(t+h)-F_{4}(t)\right\|^{2} \leq & 2\left\|\int_{0}^{t}[S(t+h-s)-S(t-s)] \sigma\left(s, x_{s}\right) d W(s)\right\|^{2} \\
& +2\left\|\int_{t}^{t+h} S(t+h-s) \sigma\left(s, x_{s}\right) d W(s)\right\|^{2} \\
\leq & 2 C_{p} C_{\sigma}^{2} \int_{0}^{t}\|S(t+h-s)-S(t-s)\|^{2}\|x\|_{s}^{2} d s \\
& +2 C_{p} C_{\sigma}^{2} \int_{t}^{t+h}\|S(t+h-s)\|^{2}\|x\|_{s}^{2} d s \\
\rightarrow & 0 \text { as }|h| \rightarrow 0
\end{aligned}
$$

Similarly,

$$
\begin{aligned}
\mathbb{E}\left\|F_{5}(t+h)-F_{5}(t)\right\|^{2} \leq & 2 \mathbb{E}\left\|\int_{0}^{t} \int_{\mathcal{U}}[S(t+h-s)-S(t-s)] h\left(s, x_{s}, u\right) \widetilde{N}(d s, d u)\right\|^{2} \\
& +2 \mathbb{E}\left\|\int_{t}^{t+h} \int_{\mathcal{U}} S(t+h-s) h\left(s, x_{s}, u\right) \tilde{N}(d s, d u)\right\|^{2} \\
\leq & 2 C_{h}\left[\mathbb{E} \int_{0}^{t} \int_{\mathcal{U}}\|S(t+h-s)-S(t-s)\|^{2}\|x\|_{s}^{2} v(d u) d s\right. \\
& \left.+\mathbb{E}\left(\int_{0}^{t} \int_{\mathcal{U}}\|S(t+h-s)-S(t-s)\|^{2}\|x\|_{s}^{4} v(d u) d s\right)^{\frac{1}{2}}\right] \\
& +2 C_{h}\left[\mathbb{E} \int_{t}^{t+h} \int_{\mathcal{U}}\|S(t+h-s)\|^{2}\|x\|_{s}^{2} v(d u) d s\right. \\
& \left.+\mathbb{E}\left(\int_{t}^{t+h} \int_{\mathcal{U}}\|S(t+h-s)\|^{2}\|x\|_{s}^{4} v(d u) d s\right)^{\frac{1}{2}}\right] \\
\rightarrow & 0 \quad \text { as }|h| \rightarrow 0
\end{aligned}
$$


For $F_{6}$, using assumptions $\left(\mathrm{A}_{1}\right)$ and $\left(\mathrm{A}_{4}\right)$, we have

$$
\begin{aligned}
\mathbb{E}\left\|F_{6}(t+h)-F_{6}(t)\right\|^{2} \leq & 2 \mathbb{E} \| \sum_{0<t_{j}<t}\left[S\left(t+h-t_{j}\right)-S\left(t-t_{j}\right)\right]\left(I_{j}\left(x\left(t_{j}\right)\right) \|^{2}\right. \\
& +2 \mathbb{E} \| \sum_{t<t_{j}<t+h} S\left(t+h-t_{j}\right)\left(I_{j}\left(x\left(t_{j}\right)\right) \|^{2}\right. \\
\leq & 2 \sum_{0<t_{j}<t} \mathbb{E}\left\|S\left(t+h-t_{j}\right)-S\left(t-t_{j}\right)\right\|^{2}\left[q_{j}^{2} \mathbb{E}\left\|x\left(t_{j}\right)\right\|^{2}\right] \\
& +2 \sum_{t<t_{j}<t+h} \mathbb{E}\left\|S\left(t+h-t_{j}\right)\right\|^{2}\left[q_{j}^{2} \mathbb{E}\left\|x\left(t_{j}\right)\right\|^{2}\right] \\
\rightarrow & 0 \quad \text { as }|h| \rightarrow 0 .
\end{aligned}
$$

Hence, the above arguments imply that $t \rightarrow \pi(x(t))$ is càdlàg on $[0, T]$ a.s.

Step (ii): We shall verify that $\pi\left(S_{T}\right) \subset \mathcal{B}_{T}$, let $x \in \mathcal{B}_{T}, t \in[0, T]$.

From Hölder's inequality,

$$
\begin{aligned}
\mathbb{E}\|\pi(x(t))\|^{2} \leq & 7 \mathbb{E}\|S(t)[\phi(0)+g(0, \phi)]\|^{2}+7 \mathbb{E}\left\|g\left(t, x_{t}\right)\right\|^{2} \\
& +7 \mathbb{E}\left\|\int_{0}^{t} A S(t-s) g\left(s, x_{s}\right) d t\right\|^{2}+7 \mathbb{E}\left\|\int_{0}^{t} S(t-s) f\left(s, x_{s}\right) d t\right\|^{2} \\
& +7 \mathbb{E}\left\|\int_{0}^{t} S(t-s) \sigma\left(s, x_{s}\right) d W(s)\right\|^{2} \\
& +7 \mathbb{E}\left\|\int_{0}^{t} \int_{\mathcal{U}} S(t-s) h\left(s, x_{s}, u\right) \tilde{N}(d s, d u)\right\|^{2} \\
& +7\left\|\sum_{0<t_{j}<t} S\left(t-t_{j}\right) I_{j}\left(x\left(t_{j}\right)\right)\right\|^{2} \\
= & 7 \sum_{i=1}^{7} F_{i} .
\end{aligned}
$$

We now estimate $F_{i}, i=1,2, \ldots, 7$. By assumption $\mathrm{A}_{2}-(2 a)$, we have

$$
\begin{aligned}
F_{1} & \leq 2\left[\mathbb{E}\|S(t) \phi(0)\|^{2}+\mathbb{E}\|S(t) g(0, \phi)\|^{2}\right] \\
& \leq 2 M^{2}\left[1+C_{g}^{2}\left\|(-A)^{-\beta}\right\|^{2}\right] \mathbb{E}\|\phi\|^{2} .
\end{aligned}
$$

Applying Hölder's inequality and $\mathrm{A}_{2}-(2 a)$, we have

$$
F_{2} \leq\left\|(-A)^{-\beta}\right\|^{2} C_{g}^{2} \mathbb{E}\|x\|_{t}^{2}
$$

and

$$
\begin{aligned}
F_{3} & \leq \mathbb{E} \int_{0}^{t}\left\|(-A)^{1-\beta} S(t-s)(-A)^{\beta} g\left(t, x_{t}\right)\right\|^{2} d s \\
& \leq M^{2} t\left\|(-A)^{1-\beta}\right\|^{2} C_{g}^{2} \int_{0}^{t} \mathbb{E}\|x\|_{s}^{2} d s .
\end{aligned}
$$


By Hölder's inequality and $\mathrm{A}_{1}-(1 a)$, we derive that

$$
\begin{aligned}
F_{4} & \leq 2 \mathbb{E} \int_{0}^{t}\left\|S(t-s)\left[f\left(s, x_{s}\right)-f(s, 0)\right]\right\|^{2} d s+2 \mathbb{E} \int_{0}^{t}\|S(t-s) f(s, 0)\|^{2} d s \\
& \leq 2 M^{2} t C_{f}^{2} \int_{0}^{t} \mathbb{E}\|x\|_{s}^{2} d s+2 t M^{2} \kappa_{0} \\
& \leq 2 M^{2} t\left[C_{f}^{2} \int_{0}^{t} \mathbb{E}\|x\|_{s}^{2} d s+\kappa_{0}\right] .
\end{aligned}
$$

On the other hand, applying assumption $\left(\mathrm{A}_{1}\right)-(1 b)$ and Lemma 2.2, we get, for some positive constant $C_{p}$,

$$
\begin{aligned}
F_{5} & \leq C_{p}\|S(t)\|^{2} \mathbb{E} \int_{0}^{t}\left\|\sigma\left(s, x_{s}\right)-\sigma(s, 0)+\sigma(s, 0)\right\|^{2} d s \\
& \leq 2 C_{p} M^{2}\left[C_{\sigma}^{2} \mathbb{E} \int_{0}^{t}\|x\|_{s}^{2} d s+\kappa_{0} t\right] .
\end{aligned}
$$

Employing assumption $\left(\mathrm{A}_{1}\right)-(1 c)$ and Lemma 2.2 in [13], we obtain

$$
\begin{aligned}
F_{6} \leq & M^{2}\left[\mathbb{E} \int_{0}^{t} \int_{\mathcal{U}}\left\|h\left(s, x_{s}, u\right)\right\|^{2} v(d u) d s+\mathbb{E}\left(\int_{0}^{t} \int_{\mathcal{U}}\left\|h\left(s, x_{s}, u\right)\right\|^{4} v(d u) d s\right)^{\frac{1}{2}}\right] \\
\leq & M^{2}\left[\mathbb{E} \int_{0}^{t} \int_{\mathcal{U}}\left\|h\left(s, x_{s}, u\right)-h(s, 0, u)+h(s, 0, u)\right\|^{2} v(d u) d s\right. \\
& \left.+\mathbb{E}\left(\int_{0}^{t} \int_{\mathcal{U}}\left\|h\left(s, x_{s}, u\right)\right\|^{4} v(d u) d s\right)^{\frac{1}{2}}\right] \\
\leq & 2 M^{2}\left[C_{h}^{2} \int_{0}^{t} \mathbb{E}\|x\|_{s}^{2} d s+\kappa_{0} t\right]+M^{2} C_{h}^{2} \int_{0}^{t} \mathbb{E}\|x\|_{s}^{2} d s \\
\leq & 3 M^{2} C_{h}^{2} \int_{0}^{t} \mathbb{E}\|x\|_{s}^{2} d s+2 M^{2} \kappa_{0} t .
\end{aligned}
$$

From Hölder's inequality and assumption $\left(\mathrm{A}_{4}\right)$, we have

$$
\begin{aligned}
F_{7} & \leq 2 \mathbb{E} \sum_{j=1}^{\infty}\left[\left\|S\left(t-t_{j}\right) I_{j}\left(x\left(t_{j}\right)\right)-I_{j}(0)\right\|^{2}+\left\|S\left(t-t_{j}\right) I_{j}(0)\right\|^{2}\right] \\
& \leq 2 M^{2}\left[\sum_{j=1}^{\infty} q_{j}^{2} \mathbb{E}\|x\|_{t}^{2}+\sum_{j=1}^{\infty} q_{j}^{2} \kappa_{0}\right] .
\end{aligned}
$$

From the above estimations, Eq. (3.2) becomes

$$
\begin{aligned}
\mathbb{E}\|\pi(x(t))\|^{2} \leq & 14 M^{2}\left[1+C_{g}^{2}\left\|(-A)^{-\beta}\right\|^{2}\right] \mathbb{E}\|\phi\|^{2}+7 C_{g}^{2}\left\|(-A)^{-\beta}\right\|^{2} \mathbb{E}\|x\|_{t}^{2} \\
& +7 M^{2} t C_{g}^{2}\left\|(-A)^{1-\beta}\right\|^{2} \int_{0}^{t} \mathbb{E}\|x\|_{s}^{2} d s \\
& +14 M^{2} t\left[C_{f}^{2} \int_{0}^{t} \mathbb{E}\|x\|_{s}^{2} d s+\kappa_{0}\right]
\end{aligned}
$$




$$
\begin{aligned}
& +14 C_{p} M^{2}\left[C_{\sigma}^{2} \int_{0}^{t} \mathbb{E}\|x\|_{s}^{2} d s+\kappa_{0} t\right] \\
& +21 M^{2} C_{h}^{2} \int_{0}^{t} \mathbb{E}\|x\|_{s}^{2} d s+14 M^{2} t \kappa_{0} \\
& +14 M^{2} \sum_{j=1}^{\infty} q_{j}^{2}\left[\mathbb{E}\|x\|_{t}^{2}+\kappa_{0}\right] \\
\leq & R_{1}+7 C_{g}^{2}\left\|(-A)^{-\beta}\right\|^{2} \mathbb{E}\|x\|_{t}^{2}+7 M^{2} t C_{g}^{2}\left\|(-A)^{1-\beta}\right\|^{2} \int_{0}^{t} \mathbb{E}\|x\|_{s}^{2} d s \\
& +7 M^{2}\left[2 t C_{f}^{2}+2 C_{p} C_{\sigma}^{2}+3 C_{h}^{2}\right] \int_{0}^{t} \mathbb{E}\|x\|_{s}^{2} d s+14 M^{2} \sum_{j=1}^{\infty} q_{j}^{2} \mathbb{E}\|x\|_{t}^{2},
\end{aligned}
$$

where $R_{1}=14 M^{2}\left[1+C_{g}^{2}\left\|(-A)^{-\beta}\right\|^{2}\right] \mathbb{E}\|\phi\|^{2}+14 M^{2}\left[2 t+C_{p} t+\sum_{j=1}^{\infty} q_{j}^{2}\right] \kappa_{0}$.

We obtain

$$
\begin{aligned}
\mathbb{E}\|\pi(x(t))\|^{2} \leq & R_{1}+7\left\{C_{g}^{2}\left\|(-A)^{-\beta}\right\|^{2}+2 M^{2} \sum_{j=1}^{\infty} q_{j}^{2}\right\} \mathbb{E}\|x\|_{t}^{2} \\
& +7 M^{2}\left[t C_{g}^{2}\left\|(-A)^{1-\beta}\right\|^{2}+2 t C_{f}^{2}+2 C_{p} C_{\sigma}^{2}+3 C_{h}^{2}\right] \int_{0}^{t} \mathbb{E}\|x\|_{s}^{2} d s .
\end{aligned}
$$

Therefore

$$
\begin{aligned}
\sup _{0 \leq s \leq T} \mathbb{E}\|\pi(x(t))\|^{2} & \leq R_{1}+R_{2} \sup _{-\tau \leq t \leq T} \mathbb{E}\|x\|_{t}^{2}+R_{3} \int_{0}^{t} \sup _{-\tau \leq s \leq T} \mathbb{E}\|x\|_{s}^{2} d s \\
& \leq R_{1}+R_{2} \sup _{-\tau \leq t \leq T} \mathbb{E}\|x\|_{t}^{2}+R_{3} t \sup _{-\tau \leq t \leq T} \mathbb{E}\|x\|_{t}^{2} \\
& \leq R_{1}+R_{4} \sup _{-\tau \leq t \leq T} \mathbb{E}\|x\|_{t}^{2},
\end{aligned}
$$

where

$$
\begin{aligned}
& R_{2}=7\left\{C_{g}^{2}\left\|(-A)^{-\beta}\right\|^{2}+2 M^{2} \sum_{j=1}^{\infty} q_{j}^{2}\right\} \\
& R_{3}=7 M^{2}\left[t C_{g}^{2}\left\|(-A)^{1-\beta}\right\|^{2}+2 t C_{f}^{2}+2 C_{p} C_{\sigma}^{2}+3 C_{h}^{2}\right] \\
& R_{4}=R_{2}+R_{3} \cdot t .
\end{aligned}
$$

Since $\pi(x)=\phi$ on $[-\tau, 0]$, it follows that

$$
\mathbb{E} \sup _{-\tau \leq s \leq T}\|\pi(x(s))\|^{2}<\infty
$$

This proves the boundedness of $\pi \mathcal{B}_{T}$.

Step (iii): Next, we will verify that $\pi$ is a contraction mapping in $\mathcal{B}_{T_{1}}$ with some $T_{1} \leq T$ to be specified later. 
Let $x, y \in \mathcal{B}_{T}$. Based on this simple inequality $(x+y+z)^{2} \leq \frac{1}{k} x^{2}+\frac{2}{1-k} y^{2}+\frac{2}{1-k} z^{2}$ and recalling that $k: c_{g}\left\|(-A)^{-\beta}\right\|<1$, for $t \in[0, T]$,

$$
\begin{aligned}
\mathbb{E}\|\pi(x(t))-\pi(y(t))\|^{2} \leq & \frac{1}{k} \mathbb{E}\left\|(-A)^{-\beta}\right\|^{2}\left\|(-A)^{\beta} g\left(t, x_{t}\right)-g\left(t, y_{t}\right)\right\|^{2} \\
& +\frac{5}{1-k} \mathbb{E}\left\|\int_{0}^{t}(-A)^{1-\beta} S(t-s)(-A)^{\beta}\left[g\left(s, x_{s}\right)-g\left(s, y_{s}\right)\right] d s\right\|^{2} \\
& +\frac{5}{1-k} \mathbb{E}\left\|\int_{0}^{t} S(t-s)\left[f\left(s, x_{s}\right)-f\left(s, y_{s}\right)\right] d s\right\|^{2} \\
& +\frac{5}{1-k} \mathbb{E}\left\|\int_{0}^{t} S(t-s)\left[\sigma\left(s, x_{s}\right)-\sigma\left(s, y_{s}\right)\right] d W s\right\|^{2} \\
& +\frac{5}{1-k} \mathbb{E}\left\|\int_{0}^{t} \int_{\mathcal{U}} S(t-s)\left[h\left(s, x_{s}, u\right)-h\left(s, y_{s}, u\right)\right] \tilde{N}(d s, d u)\right\|^{2} \\
& +\frac{5}{1-k} \mathbb{E}\left\|\sum_{0<t_{j}<t} S\left(t-t_{j}\right)\left[I_{j}\left(x\left(t_{j}\right)\right)-I_{j}\left(y\left(t_{j}\right)\right)\right]\right\|^{2} .
\end{aligned}
$$

By using Holder's inequality, Lemma 2.2 together with assumptions $\left(A_{1}\right),\left(A_{2}\right)$, and $\left(A_{4}\right)$, we get

$$
\begin{aligned}
\mathbb{E} \| & (x(t))-\pi(y(t)) \|^{2} \\
\leq & k \mathbb{E}\|x-y\|_{t}^{2} \\
& +\frac{5}{1-k} M_{1-\beta}^{2} C_{g}^{2}\left(\frac{t^{2 \beta-1}}{2 \beta-1}\right) \int_{0}^{t} \mathbb{E}\|x-y\|_{s}^{2} d s \\
& +\frac{5}{1-k} M^{2} t C_{f}^{2} \int_{0}^{t} \mathbb{E}\|x-y\|_{s}^{2} d s+\frac{5}{1-k} M^{2} C_{\sigma}^{2} C_{p} \int_{0}^{t} \mathbb{E}\|x-y\|_{s}^{2} d s \\
& +\frac{5}{1-k} M^{2} C_{h}^{2} \int_{0}^{t} \mathbb{E}\|x-y\|_{s}^{2} d s+\frac{5}{1-k} M^{2} \sum_{j=1}^{\infty} q_{j}^{2} \mathbb{E}\|x-y\|_{t}^{2} \\
\mathbb{E} \| & (x(t))-\pi(y(t)) \|^{2} \\
\leq & k \mathbb{E}\|x-y\|_{t}^{2} \\
& +\frac{5}{1-k}\left[C_{g}^{2} M_{1-\beta}^{2}\left(\frac{t^{2 \beta-1}}{2 \beta-1}\right)+M^{2}\left(t C_{f}^{2}+C_{p} C_{\sigma}^{2}+C_{h}^{2}\right)\right] \int_{0}^{t} \mathbb{E}\|x-y\|_{s}^{2} d s \\
& +\frac{5}{1-k} M^{2} \sum_{j=1}^{\infty} q_{j}^{2} \mathbb{E}\|x-y\|_{t}^{2} .
\end{aligned}
$$

Hence, $\sup _{s \in[-\tau, T]} \mathbb{E}\|\pi(x(t))-\pi(y(t))\|^{2} \leq \gamma(t) \sup _{s \in[-\tau, T]} \mathbb{E}\|x-y\|_{s}^{2}$, where $\gamma(t)=k+$ $\frac{5}{1-k}\left[C_{g}^{2} M_{1-\beta}^{2}\left(\frac{t^{2 \beta}}{2 \beta-1}\right)+M^{2} t\left(t C_{f}^{2}+C_{p} C_{\sigma}^{2}+C_{h}^{2}\right)\right]+\frac{5 M^{2}}{1-k} \sum_{j=1}^{\infty} q_{j}^{2}$. By Eq. (3.1), we have $\gamma(0)=$ $k+\frac{5 M^{2}}{1-k} \sum_{j=1}^{\infty} q_{j}^{2}=\frac{5 M^{2} \sum_{j=1}^{\infty} q_{j}^{2}}{(1-k)^{2}}<1$. Hence, there exists $0<T_{1}<T$ such that $0<\gamma\left(T_{1}\right)<1$ and $\pi$ is a contraction mapping on $\mathcal{B}_{T_{1}}$. Therefore it is clear that it has a unique fixed point, which is a mild solution of (2.1)-(2.3). By repeating a similar process the solution can be extended to the entire interval $[-\tau, T]$ in infinitely many steps. This concludes Theorem 3.1. 


\section{Stability}

Definition 4.1 Let $x, \hat{x}$ be different mild solutions of (2.1)-(2.3) with initial values $\phi_{1}$ and $\phi_{2}$, respectively. If for all $\epsilon>0, \exists \delta>0$ such that $\mathbb{E}\|x(t)-\hat{x}(t)\|^{2} \leq \epsilon$ when $\mathbb{E}\left\|\phi_{1}-\phi_{2}\right\|^{2}<\delta$ for all $t \in[0, T]$, then $x(t)$ is said to be stable in mean square.

Theorem 4.1 Assume that any two mild solutions of (2.1)-(2.3) are $x(t)$ and $y(t)$ with initial values $\phi_{1}$ and $\phi_{2}$, respectively. Suppose that $\left(\mathrm{A}_{1}\right)-\left(\mathrm{A}_{4}\right)$ are satisfied, then the mild solution of (2.1)-(2.3) is stable in the quadratic mean.

Proof For $0 \leq t \leq T$,

$$
\begin{aligned}
& \mathbb{E}\|x(t)-y(t)\|^{2} \\
& \leq 7 \mathbb{E}\left\|S(t)\left(\left[\phi_{1}(0)-\phi_{2}(0)\right]+\left[g\left(0, \phi_{1}\right)-g\left(0, \phi_{2}\right)\right]\right)\right\|^{2}+7 \mathbb{E}\left\|g\left(t, x_{t}\right)-g\left(t, y_{t}\right)\right\|^{2} \\
&+7 \mathbb{E}\left\|\int_{0}^{t} A S(t-s)\left[g\left(s, x_{s}\right)-g\left(s, y_{s}\right)\right] d s\right\|^{2}+7 \mathbb{E}\left\|\int_{0}^{t} S(t-s)\left[f\left(s, x_{s}\right)-f\left(s, y_{s}\right)\right] d s\right\|^{2} \\
&+7 \mathbb{E}\left\|\int_{0}^{t} S(t-s)\left[\sigma\left(s, x_{s}\right)-\sigma\left(s, y_{s}\right)\right] d W(s)\right\|^{2} \\
&+7 \mathbb{E}\left\|\int_{0}^{t} \int_{\mathcal{U}} S(t-s)\left[h\left(s, x_{s}, u\right)-h\left(s, y_{s}, u\right)\right] \widetilde{N}(d s, d u)\right\|^{2} \\
&+7 \mathbb{E}\left\|\sum_{0<t_{j}<t} S\left(t-t_{j}\right)\left[I_{j}\left(x\left(t_{j}\right)\right)\right]\right\|^{2} .
\end{aligned}
$$

By using Hölder's inequality and assumptions $\left(\mathrm{A}_{1}\right),\left(\mathrm{A}_{2}\right)$, and $\left(\mathrm{A}_{4}\right)$, we derive that

$$
\begin{aligned}
\mathbb{E} \| x(t) & -y(t) \|^{2} \\
\leq & 7 M^{2}\left[1+C_{g}^{2}\left\|(-A)^{-\beta}\right\|^{2}\right] \mathbb{E}\left\|\phi_{1}-\phi_{2}\right\|^{2} \\
& +7 C_{g}^{2}\left\|(-A)^{-\beta}\right\|^{2} \mathbb{E}\|x-y\|_{t}^{2} \\
& +7 M_{1-\beta}^{2} C_{g}^{2}\left(\frac{t^{2 \beta-1}}{2 \beta-1}\right) \int_{0}^{t} \mathbb{E}\|x-y\|_{s}^{2} d s \\
& +7 M^{2} t C_{f}^{2} \int_{0}^{t} \mathbb{E}\|x-y\|_{s}^{2} d s+7 M^{2} C_{\sigma}^{2} C_{p} \int_{0}^{t} \mathbb{E}\|x-y\|_{s}^{2} d s \\
& +7 M^{2} C_{h}^{2} \int_{0}^{t} \mathbb{E}\|x-y\|_{s}^{2} d s+7 M^{2} \sum_{j=1}^{\infty} q_{j}^{2} \mathbb{E}\|x-y\|_{t}^{2} \\
\leq & 7 M^{2}\left[1+C_{g}^{2}\left\|(-A)^{-\beta}\right\|^{2}\right] \mathbb{E}\left\|\phi_{1}-\phi_{2}\right\|^{2} \\
& +7\left[C_{g}^{2}\left\|(-A)^{-\beta}\right\|^{2}+M^{2} \sum_{j=1}^{\infty} q_{j}^{2}\right] \mathbb{E}\|x-y\|_{t}^{2} \\
& \left.+7\left[M_{1-\beta}^{2} C_{g}^{2}\left(\frac{t^{2 \beta-1}}{2 \beta-1}\right)+M^{2}\left(t C_{f}^{2}+C_{p} C_{\sigma}^{2}+C_{h}^{2}\right)\right)\right] \int_{0}^{t} \mathbb{E}\|x-y\|_{s}^{2} d s .
\end{aligned}
$$


It follows that

$$
\begin{aligned}
\sup _{t \in[\tau, T]} \mathbb{E}\|x-y\|_{t}^{2} \\
\leq \frac{7 M^{2}\left[1+\left\|(-A)^{-\beta}\right\|^{2} C_{g}^{2}\right]}{1-Q} \mathbb{E}\left\|\phi_{1}-\phi_{2}\right\|^{2} \\
\quad+\frac{7\left[M_{1-\beta}^{2} C_{g}^{2}\left(\frac{t^{2 \beta-1}}{2 \beta-1}\right)+M^{2}\left(t C_{f}^{2}+C_{p} C_{\sigma}^{2}+C_{h}^{2}\right)\right]}{1-Q} \int_{0}^{t} \sup _{t \in[\tau, T]} \mathbb{E}\|x-y\|_{s}^{2} d s,
\end{aligned}
$$

where $Q=7\left[C_{g}^{2}\left\|(-A)^{-\beta}\right\|^{2}+M^{2} \sum_{j=1}^{\infty} q_{j}^{2}\right]$.

By applying Gronwall's inequality, we have

$$
\begin{aligned}
\sup _{t \in[\tau, T]} \mathbb{E}\|x-y\|_{t}^{2} \leq & \frac{7 M^{2}\left[1+\left\|(-A)^{-\beta}\right\|^{2} C_{g}^{2}\right]}{1-Q} \mathbb{E}\left\|\phi_{1}-\phi_{2}\right\|^{2} \\
& \times \exp \left(\frac{7\left[M_{1-\beta}^{2} C_{g}^{2}\left(\frac{t^{2 \beta-1}}{2 \beta-1}\right)+M^{2}\left(t C_{f}^{2}+C_{p} C_{\sigma}^{2}+C_{h}^{2}\right)\right]}{1-Q}\right) \\
\leq & \\
\leq & \mathbb{E}\left\|\phi_{1}-\phi_{2}\right\|^{2},
\end{aligned}
$$

where $\wp=\frac{7 M^{2}\left[1+\left\|(-A)^{-\beta}\right\|^{2} C_{g}^{2}\right]}{1-Q} \exp \left(\frac{7\left[M_{1-\beta}^{2} C_{g}^{2}\left(\frac{t^{2 \beta-1}}{2 \beta-1}\right)+M^{2}\left(t C_{f}^{2}+C_{p} C_{\sigma}^{2}+C_{h}^{2}\right)\right]}{1-Q}\right)$.

Now, given $\epsilon>0$, choose $\delta=\frac{\epsilon}{\wp}$ such that $\mathbb{E}\left\|\phi_{1}-\phi_{2}\right\|^{2}<\delta$. Then

$$
\sup _{t \in[\tau, T]} \mathbb{E}\|x-y\|_{t}^{2} \leq \epsilon
$$

This concludes Theorem 4.1.

\section{Exponential stability}

A system is defined to be exponentially stable if the system response decays exponentially towards zero as time approaches infinity.

For example, consider that a system, marble ball in a ladle, when undisturbed will occupy the lowest point in the ladle. But when the ball is subjected to a push, it will exhibit a diminishing sinusoidal oscillation and eventually resettle in the bottom of the ladder. Also, the system is said to be marginally stable when the ball is away from the bottom of the ladle when a constant force equal to its weight is applied. But when the ball is given a big push, it will fall away from the ladle and stop when it reaches the ground. Therefore it is proper to state that the system is exponentially stable for a range of inputs.

Definition 5.1 System (2.1) - (2.3) is said to be exponentially stable in the quadratic mean if there exist positive constant $C_{1}$ and $\lambda>0$ such that

$$
E\|x(t)\|^{2} \leq C_{1} E\|\varphi\|^{2} e^{-\lambda\left(t-t_{0}\right)}, \quad t \geq t_{0}
$$

We assume that $f(t, 0)=\sigma(t, 0)=h(t, 0, u)=0$ for all $t \geq 0, u \in \mathcal{U}$. So that system (2.1)-(2.3) admits a trivial solution. We further need the following assumptions.

$\left(\mathrm{A}_{5}\right)\|S(t)\| \leq M e^{-\lambda\left(t-t_{0}\right)}, t \geq t_{0}$, where $M \geq 1, \lambda>0$. 
$\left(\mathrm{A}_{6}\right)$ There exist nonnegative real numbers $E_{1}, E_{2}, E_{3}, E_{4} \geq 0$ and continuous functions $\delta_{1}, \delta_{2}, \delta_{3}, \delta_{4}:[0,+\infty) \rightarrow \mathbb{R}_{+}$such that, for all $t \geq 0$ and $x, y \in X$,

$$
\begin{aligned}
\text { (i) } & \left\|f\left(t, x_{t}\right)\right\|^{2} \leq E_{1}\|x\|_{t}^{2}+\delta_{1}(t), \\
\text { (ii) } & \left\|(-A)^{\beta} g\left(t, x_{t}\right)\right\|^{2} \leq E_{2}\|x\|_{t}^{2}+\delta_{2}(t), \\
\text { (iii) } & \left\|\sigma\left(t, x_{t}\right)\right\|^{2} \leq E_{3}\|x\|_{t}^{2}+\delta_{3}(t), \\
\text { (iv) } & \int_{\mathcal{U}}\left\|h\left(t, x_{t}, u\right)\right\|^{2} v(d u) \vee\left(\int_{\mathcal{U}}\left\|h\left(t, x_{t}, u\right)\right\|^{4} v(d u)\right)^{\frac{1}{2}} \leq E_{4}\|x\|_{t}^{2}+\delta_{4}(t) .
\end{aligned}
$$

$\left(\mathrm{A}_{7}\right)$ There exist nonnegative real numbers $P_{j} \geq 0, j=1,2,3,4$, such that $\delta_{j}(t) \leq P_{j} e^{-\lambda t}$, $\forall t \geq 0, j=1,2,3,4$.

Theorem 5.1 Assume that $\left(\mathrm{A}_{4}\right)-\left(\mathrm{A}_{7}\right)$ and the following inequality holds:

$$
\begin{aligned}
& \frac{6\left\{\left[\lambda^{1-2 \beta} 2^{2(1-\beta)} M_{1-\beta}^{2} M^{2} \Gamma(2 \beta-1) E_{2} / \lambda\right]+M^{2}\left[E_{1}^{2}+C_{p} E_{3}^{2}+E_{4}^{2}\right] / \lambda^{2}+M^{2} \sum_{k=1}^{\infty} q_{j}^{2}\right\}}{(1-k)^{2}} \\
& <1,
\end{aligned}
$$

where $k=\sqrt{E_{2}}\left\|(-A)^{-\beta}\right\|$. Then the mild solution of system (2.1)-(2.3) is exponentially stable in the mean square moment.

Proof From inequality (5.1), it is possible to find a small positive quantity $\epsilon$ such that

$$
k+\frac{6 \lambda^{1-2 \beta} 2^{2(1-\beta)} M_{1-\beta}^{2} M^{2} \Gamma(2 \beta-1) E_{2}}{(\lambda-\epsilon)(1-k)}+\frac{6 M^{2}\left[E_{1}^{2}+C_{p} E_{3}^{2}+E_{4}^{2}\right]}{\lambda(\lambda-\epsilon)(1-k)}+\frac{6 M^{2} \sum_{k=1}^{\infty} q_{j}^{2}}{(1-k)}<1 .
$$

Let $\eta=\lambda-\epsilon$ and $x(t)$ be the mild solution of (2.1)-(2.3).

For $t \geq 0$,

$$
\begin{aligned}
\mathbb{E}\|x(t)\|^{2} \leq & \frac{1}{k} \mathbb{E}\left\|g\left(t, x_{t}\right)\right\|^{2}+\frac{6}{1-k} \mathbb{E}\left\{\|S(t)[\phi(0)+g(0, \phi)]\|^{2}\right. \\
& +\left\|\int_{0}^{t} A S(t-s) g\left(s, x_{s}\right) d s\right\|^{2}+\left\|\int_{0}^{t} S(t-s) f\left(s, x_{s}\right) d s\right\|^{2} \\
& +\left\|\int_{0}^{t} S(t-s) \sigma\left(s, x_{s}\right) d W(s)\right\|^{2}+\left\|\int_{0}^{t} \int_{\mathcal{U}} S(t-s) h\left(s, x_{s}, u\right) \widetilde{N}(d s, d u)\right\|^{2} \\
& \left.+\left\|\sum_{0<t_{j}<t} S\left(t-t_{j}\right) I_{j}\left(x\left(t_{j}\right)\right)\right\|^{2}\right\} \\
\leq & \sum_{j=1}^{7} F_{j}(t) .
\end{aligned}
$$


By conditions $\left(\mathrm{A}_{6}\right)$ and $\left(\mathrm{A}_{7}\right)$, we obtain

$$
\begin{aligned}
F_{1}(t) & =\frac{1}{k} \mathbb{E}\left\|(-A)^{-\beta}(-A)^{\beta} g\left(t, x_{t}\right)\right\|^{2} \\
& \leq \frac{\left\|(-A)^{-\beta}\right\|^{2}}{k}\left[E_{2}^{2} \mathbb{E}\|x\|_{t}^{2}+\delta_{2}\right] \\
& \leq k \mathbb{E}\|x\|_{t}^{2}+K_{1} e^{-\eta t} \quad \text { where } K_{1}=\frac{\left\|(-A)^{-\beta}\right\|^{2} P_{2}}{k} .
\end{aligned}
$$

Using assumptions $\left(\mathrm{A}_{5}\right),\left(\mathrm{A}_{6}\right)$, and $\left(\mathrm{A}_{7}\right)$, we have

$$
\begin{aligned}
F_{2}(t) & \leq \frac{12}{1-k}\left[\mathbb{E}\|S(t) \phi(0)\|^{2}+\mathbb{E}\|S(t) g(0, \phi)\|^{2}\right] \\
& \leq \frac{12 M^{2}}{1-k} e^{-2 \lambda t} \mathbb{E}\|\phi(0)\|^{2}+\frac{12 M^{2}}{1-k} e^{-2 \lambda t}\left\|(-A)^{-\beta}\right\|^{2}\left[E_{2} \mathbb{E}\|\phi\|^{2}+P_{2}\right] \\
& \leq K_{2} e^{-\eta t}, \\
\text { where } K_{2} & =\frac{12 M^{2}}{1-k}\left\{\mathbb{E}\|\phi(0)\|^{2}+\left\|(-A)^{-\beta}\right\|^{2}\left[E_{2} \mathbb{E}\|\phi\|^{2}+P_{2}\right]\right\} .
\end{aligned}
$$

Applying assumptions $\left(A_{5}\right),\left(A_{6}\right)$, and $\left(A_{7}\right)$ together with Lemma 2.1 and Hölder's inequality, we get

$$
\begin{aligned}
F_{3}(t) & =\frac{6}{1-k} \mathbb{E}\left\|\int_{0}^{t}(-A)^{1-\beta} S\left(\frac{t-s}{2}\right) S\left(\frac{t-s}{2}\right)(-A)^{\beta} g\left(s, x_{s}\right) d s\right\|^{2} \\
& \leq \frac{6}{1-k} \int_{0}^{t} \frac{M_{1-\beta}^{2} e^{-\lambda(t-s)}}{\left(\frac{t-s}{2}\right)^{2(1-\beta) d s}} \int_{0}^{t} M^{2} e^{-\lambda(t-s)} \mathbb{E}\left\|(-A)^{\beta} g\left(s, x_{s}\right)\right\|^{2} d s \\
& \leq \frac{6 \lambda^{1-2 \beta} 2^{2(1-\beta)} M_{1-\beta}^{2} M^{2} \Gamma(2 \beta-1)}{1-k} \int_{0}^{t} e^{-\lambda(t-s)}\left[E_{2} \mathbb{E}\|x\|_{s}^{2}+\delta_{2}(s)\right] d s \\
& \leq \frac{6 \lambda^{1-2 \beta} 2^{2(1-\beta)} M_{1-\beta}^{2} M^{2} \Gamma(2 \beta-1) E_{2}}{1-k} \int_{0}^{t} e^{-\lambda(t-s)} \mathbb{E}\|x\|_{s}^{2} d s+K_{3} e^{-\eta t},
\end{aligned}
$$

where $\Gamma$ is the usual gamma function and $K_{3}=\frac{6 \lambda^{1-2 \beta} 2^{2(1-\beta)} M^{2} M_{1-\beta}^{2} \Gamma(2 \beta-1)}{1-k} \frac{P_{2}}{\lambda-\eta}$.

Again, using $\left(\mathrm{A}_{5}\right)-\left(\mathrm{A}_{7}\right)$ and Hölder's inequality, we get

$$
\begin{aligned}
F_{4}(t) & =\frac{6}{1-k} \mathbb{E}\left(\int_{0}^{t} S(t-s) e^{-\lambda(t-s)}\left\|f\left(s, x_{s}\right)\right\| d s\right)^{2} \\
& \leq \frac{6 M^{2}}{1-k} \int_{0}^{t} e^{-\lambda(t-s)} d s \int_{0}^{t} e^{-\lambda(t-s)}\left[E_{1}^{2} \mathbb{E}\|x\|_{s}^{2}+\delta_{1}(s)\right] d s \\
& \leq \frac{6 M^{2} E_{1}^{2}}{(1-k) \lambda} \int_{0}^{t} e^{-\lambda(t-s)} \mathbb{E}\|x\|_{s}^{2} d s+K_{4} e^{-\eta t},
\end{aligned}
$$

where $K_{4}=\frac{6 M^{2}}{\lambda(1-k)} \frac{P_{1}}{\lambda-\eta}$. Similarly, for the term $F_{5}$,

$$
\begin{aligned}
F_{5}(t) & \leq \frac{6}{1-k}\left(\mathbb{E}\left\|\int_{0}^{t} S(t-s) \sigma\left(s, x_{s}\right) d W(s)\right\|\right)^{2} \\
& \leq \frac{6 M^{2}}{1-k} C_{p}\left(\int_{0}^{t} e^{-\lambda(t-s)} \mathbb{E}\left\|\sigma\left(s, x_{s}\right)\right\| d s\right)^{2}
\end{aligned}
$$




$$
\begin{aligned}
\leq & \frac{6}{1-k} C_{p} M^{2} \int_{0}^{t} e^{-\lambda(t-s)} d s \\
& \times \int_{0}^{t} e^{-\lambda(t-s)} d s\left[E_{3} \mathbb{E}\|x\|_{s}^{2}+\delta_{3}(s)\right] d s \\
\leq & \frac{6 C_{p} M^{2} E_{3}}{\lambda(1-k)} \int_{0}^{t} e^{-\lambda(t-s)} \mathbb{E}\|x\|_{s}^{2} d s+K_{5} e^{-\eta t},
\end{aligned}
$$

where $K_{5}=\frac{6 C_{p} M^{2}}{\lambda(1-k)} \frac{P_{3}}{\lambda-\eta}$. By assumptions $\left(\mathrm{A}_{5}\right)-\left(\mathrm{A}_{7}\right)$ together with Lemma 2.3 , we have

$$
\begin{aligned}
F_{6}(t) \leq & \frac{6}{1-k} \mathbb{E}\left(\left\|\int_{0}^{t} \int_{\mathcal{U}} S(t-s) h\left(s, x_{s}, u\right) \tilde{N}(d s, d u)\right\|\right)^{2} \\
\leq & \frac{6}{1-k} M^{2}\left(\int _ { 0 } ^ { t } e ^ { - 2 \lambda ( t - s ) } \left[\int_{\mathcal{U}} \mathbb{E}\left\|h\left(s, x_{s}, u\right)\right\|^{2} v(d u)\right.\right. \\
& \left.\left.+\left(\int_{\mathcal{U}} \mathbb{E}\left\|h\left(s, x_{s}, u\right)\right\|^{4} v(d u)\right)^{\frac{1}{2}}\right] d s\right) \\
\leq & \frac{M^{2}}{\lambda(1-k)} \int_{0}^{t} e^{-\lambda(t-s)}\left[E_{4} \mathbb{E}\|x\|_{s}^{2}+\delta_{4}(s)\right] d s \\
\leq & \frac{6 M^{2}}{\lambda(1-k)} E_{4} \int_{0}^{t} e^{-\lambda(t-s)} \mathbb{E}\|x\|_{s}^{2} d s+K_{6} e^{-\eta t},
\end{aligned}
$$

where $K_{6}=\frac{6 M^{2}}{\lambda(1-k)} \frac{P_{4}}{\lambda-\eta}$. By applying assumption $\left(\mathrm{A}_{4}\right)$, one can get

$$
\begin{aligned}
F_{7}(t) & \leq \frac{6 M^{2}}{1-k} \sum_{j=1}^{\infty} q_{j}^{2} e^{-2 \lambda\left(t-t_{j}\right)} \mathbb{E}\left\|x\left(t_{j}\right)\right\|^{2} \\
& \leq \frac{6 M^{2}}{1-k} \sum_{j=1}^{\infty} q_{j}^{2} e^{-\eta\left(t-t_{j}\right)} \mathbb{E}\left\|x\left(t_{j}\right)\right\|^{2} .
\end{aligned}
$$

The above inequalities together with Lemma 2.3 imply that

$$
\mathbb{E}\|x(t)\|^{2} \leq \gamma e^{-\eta t} \quad \text { for } t \in[-\tau, 0]
$$

and

$$
\begin{aligned}
\mathbb{E}\|x(t)\|^{2} \leq & \gamma e^{-\eta t}+k \sup _{-\tau \leq u \leq 0} \mathbb{E}\|x(t+u)\|^{2} \\
& +\widetilde{k} \int_{0}^{t} e^{-\eta(t-s)} \sup _{-\tau \leq u \leq 0} \mathbb{E}\|x(t+u)\|^{2} d s \\
& +\sum_{j=1}^{\infty} e^{-\eta\left(t-t_{j}\right)} \mathbb{E}\left\|x\left(t_{j}\right)\right\|^{2} \quad \text { for } t \geq 0 .
\end{aligned}
$$

Here $\gamma=\max \left(\sum_{i=1}^{6} K_{i}, \sup _{-\tau \leq u \leq 0} \mathbb{E}\|\phi(u)\|^{2}\right)$ and

$$
\widetilde{k}=\frac{6 \lambda^{1-2 \beta} 2^{2(1-\beta)} M^{2} M_{1-\beta}^{2} \Gamma(2 \beta-1) E_{2}}{1-k}+\frac{6 M^{2}\left[E_{1}^{2}+C_{p} E_{3}^{2}+E_{4}^{2}\right]}{\lambda(1-k)}
$$


since $k+\frac{\widetilde{k}}{\eta}+\sum_{i=1}^{\infty} d_{i}^{2}<1$, and from Lemma 2.3 there exist constants $K>0$ and $\theta>0$ such that $\mathbb{E}\|x(t)\|^{2} \leq K e^{-\theta t}, \forall t \geq-\tau$. This ensures the exponential stability of the mild solution in mean square. Hence the proof.

Remark 5.1 If the impulsive term $\Delta\left(x\left(t_{j}\right)\right)=I_{j}(\cdot)=0, j=1,2, \ldots$, then $(2.1)-(2.3)$ takes the following form:

$$
\begin{aligned}
& d\left[x(t)+g\left(t, x_{t}\right)\right]= {\left[A x(t)+f\left(t, x_{t}\right)\right] d t+\sigma\left(t, x_{t}\right) d W(t) } \\
&+\int_{\mathcal{U}} h\left(t, x_{t}, u\right) \tilde{N}(d t, d u), \quad 0 \leq t \leq T, \\
& x(t)=\phi(t), \quad-\tau \leq t \leq 0,
\end{aligned}
$$

where $C=C([-\tau, 0] ; X)$ denotes the family of almost surely bounded and continuous functions $\phi$ from $[-\tau, 0]$ into $X$ and, as usual, with $\|\phi\|_{c}=\sup _{\theta \in[-\tau, 0]}\|\phi(\theta)\|$. Also, if we assume that all the functions are defined the same as earlier, then by the same procedure as in Theorem 5.1, we may deduce the next corollary.

Corollary 5.2 Suppose that $\left(\mathrm{A}_{1}\right)-\left(\mathrm{A}_{3}\right)$ and $\left(\mathrm{A}_{5}\right)-\left(\mathrm{A}_{7}\right)$ are satisfied, then the mild solution of (2.1)-(2.3) is exponentially stable in the mean square moment if the following inequality holds:

$$
\frac{5\left\{\left[\lambda^{1-2 \beta} 2^{2(1-\beta)} M_{1-\beta}^{2} M^{2} \Gamma(2 \beta-1) E_{2} / \lambda\right]+M^{2}\left[E_{1}^{2}+C_{p} E_{3}^{2}+E_{4}^{2}\right] / \lambda^{2}\right\}}{(1-k)^{2}}<1 .
$$

\section{Conclusion}

In this article, the existence and uniqueness results for neutral impulsive stochastic functional differential equations with Poisson jumps have been derived using fixed point approach. Also, sufficient conditions are derived for the continuous dependence of solutions on the initial value by means of the corollary of Bihari's inequality. Finally, the exponential stability of mild solutions for neutral impulsive stochastic functional differential equations with Poisson jumps is investigated based on the impulsive integral inequality. This will motivate the future research work such as the study of controllability and stability in distribution for neutral impulsive stochastic functional differential equations with Poisson jumps.

\section{Acknowledgements}

The authors would like to thank the reviewers for their constructive comments in upgrading the article.

Funding

This work was partially supported by UGC, India (F MRP-5820/15(SERO/UGC)).

Availability of data and materials

Not applicable.

Competing interests

The authors declare that they have no competing interests.

Authors' contributions

All authors read and approved the final manuscript.

\section{Author details}

'Department of Mathematics, PSG College of Arts \& Science, Coimbatore, India. ²Department of Mathematics, Cankaya University, Ankara, Turkey. ${ }^{3}$ Institute of Space Sciences, Magurele-Bucharest, Romania. ${ }^{4}$ Amrita Vishwa Vidyapeetham, Coimbatore, India. 


\section{Publisher's Note}

Springer Nature remains neutral with regard to jurisdictional claims in published maps and institutional affiliations.

Received: 26 February 2018 Accepted: 21 July 2018 Published online: 24 August 2018

\section{References}

1. Anguraj, A., Vinodkumar, A.: Existence,uniqueness and stability results of impulsive stochastic semilinear neutral functional differential equations with infinite delays. Electron. J. Qual. Theory Differ. Equ. 2009, 67 (2009)

2. Arthi, G., Park, J.H., Jung, H.Y.: Existence and exponential stability for neutral stochastic integro differential equations with impulses driven by fractional Brownian motion. Commun. Nonlinear Sci. Numer. Simul. (2015). https://doi.org/10.1016/j.cnsns2015.08.014

3. Bouchard, B., Elie, R.: Discrete time approximation of decoupled forward-backward SDE with jumps. Stoch. Process. Appl. 118(1), 53-75 (2008) ([1], Appendix)

4. Boufoussi, B., Hajji, S.: Successive approximation of neutral functional stochastic differential equations with jumps. Stat. Probab. Lett. 80, 324-332 (2010)

5. Boufoussi, B., Hajji, S.: Neutral stochastic functional differential equations driven by a fractional Brownian motion in a Hilbert space. Stat. Probab. Lett. 82, 1549-1558 (2012)

6. Chen, H.: The existence and exponential stability for neutral stochastic partial differential equations with infinite delay and Poisson jumps. Indian J. Pure Appl. Math. 46(2), 197-217 (2015)

7. Chen, H., Zhu, C., Zhang, Y.: A note on exponential stability for impulsive neutral stochastic partial differential equations. Appl. Math. Comput. 227, 139-147 (2014)

8. Cui, J., Yan, L.: Successive approximation of neutral stochastic evolution equations with infinite delay and Poisson jumps. Appl. Math. Comput. 218, 6776-6784 (2012)

9. Da Prato, G., Zabczyk, J.: Stochastic Equations in Infinite Dimensions. Cambridge University Press, Cambridge (2014)

10. Feng, L., Li, S.: The pth moment asymptotic stability and exponential stability of stochastic functional differential equations with polynomial growth condition. Adv. Differ. Equ. 2014, 302 (2014)

11. Hale, J., Verduyn Lunel, S.M.: Introduction to Functional Differential Equations. Springer, New York (1993)

12. Ikeda, N., Watanabe, S.: Stochastic Differential Equations and Diffusion Processes. North-Holland, New York (1989)

13. Kolmanovskii, V.B., Myshkis, A.: Applied Theory of Functional Differential Equations. Kluwer Academic, Norwell (1992)

14. Luo, J., Liu, K.: Stability of infinite dimensional stochastic evolution equations with memory and Markovian jumps. Stoch. Process. Appl. 118, 864-895 (2008)

15. Ma, Y.-K., Arthi, G., Anthoni, M.: Exponential stability behavior of neutral stochastic integrodifferential equations with fractional Brownian motion and impulsive effects. Adv. Differ. Equ. 2018, 110 (2018)

16. Pazy, A.: Semigroups of Linear Operators and Applications to Partial Differential Equations. Applied Mathematical Sciences, vol. 44. Springer, New York (1983)

17. Pei, B., Xu, Y: Mild solutions of local non Lipschitz stochastic evolution equations with jumps. Appl. Math. Comput. 52, 80-86 (2016)

18. Sakthivel, R., Luo, J.: Asymptotic stability of nonlinear impulsive stochastic partial differential equations with infinite delays. J. Math. Anal. Appl. 356, 1-6 (2009)

19. Sun, M., Xu, M.: Exponential stability and interval stability of a class of stochastic hybrid systems driven by both Brownian motion and Poisson jumps. Physica A 487, 58-73 (2017)

20. Taniguchi, T.: Successive approximation to solutions of stochastic differential equations. J. Differ. Equ. 96, 152-169 (1992)

21. Wang, L., Cheng, T., Zhang, Q.: Successive approximation to solutions of stochastic differential equations with jumps in local non-Lipschitz conditions. Appl. Math. Comput. 225, 142-150 (2013)

22. Yang, H., Jiang, F.: Exponential stability of mild solutions to impulsive stochastic neutral partial differential equations with memory. Adv. Differ. Equ. 2013, Article ID 148 (2013)

23. Yue, C.: Neutral stochastic functional differential equations with infinite delay and Poisson jumps in the Cg space. Appl. Math. Comput. 237, 595-604 (2014)

24. Zhang, X., Zhu, C., Yuan, C.: Approximate controllability of impulsive fractional stochastic differential equations with state-dependent delay. Adv. Differ. Equ. 2015, 91 (2015)

\section{Submit your manuscript to a SpringerOpen ${ }^{\circ}$ journal and benefit from:}

- Convenient online submission

- Rigorous peer review

- Open access: articles freely available online

- High visibility within the field

- Retaining the copyright to your article

Submit your next manuscript at $\boldsymbol{~ s p r i n g e r o p e n . c o m ~}$ 